\title{
Optimal integration of wind energy with a shunt-FACTS controller for reductions in electrical power loss
}

\author{
I Made Wartana ${ }^{1}$, Ni Putu Agustini' ${ }^{2}$, Sasidharan Sreedharan ${ }^{3}$ \\ ${ }^{1,2}$ Department of Electrical Engineering, National Institute of Technology Malang, Indonesia \\ ${ }^{2}$ Department of Electrical \& Electronics Engineering, M E S College of Engineering, Kuttippuram, Kerala, India
}

\begin{abstract}
Article Info
Article history:

Received Dec 10, 2020

Revised Feb 12, 2021

Accepted Apr 12, 2021

Keywords:

Maximizing load bus Non-dominated sorting genetic algorithm II

Power loss

Small-signal stability

Static var compensator

Wind turbine generator

ABSTRACT

The integration of distributed generators (DGs) with flexible alternating current transmission systems (FACTS) can improve the performance of the grid system. In this study, we determine the location and optimal size of one type of DG, based on wind energy, with a shunt-FACTS control device called a static var compensator (SVC). The voltage profile is increase and the power loss reduced due to an improvement in performance from the maximizing load bus system scenario. Newton-Raphson power flow with a wind turbine generator (WTG) and SVC are formulated as a multi-objective problem called MLB system and minimizing system power loss (Ploss) by satisfying various system constraints, namely the loading limits, generation limits, voltage limits, and the small-signal stability. A variant of the genetic algorithm, called the non-dominated sorting genetic algorithm II (NSGA-II), is used to solve these conflicting multi-objective optimization problems. Modifications to the IEEE 14-bus standard and practical test system integrated to the WTG and SVC in the PSAT software are used as a test system. The simulation results indicate that the optimal allocation of the WTG and SVC, determined using the proposed technique, results in improved system performance, since all the specified constraints are met.
\end{abstract}

This is an open access article under the CC BY-SA license.

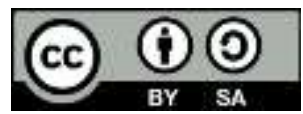

\section{Corresponding Author:}

I Made Wartana

Department of Electrical Engineering

National Institute of Technology (ITN) Malang, Indonesia

$2^{\text {nd }}$ Campus, Jl. Raya Karanglo Km. 2, Malang 65143

Tel. +62 (0) 341 - 551431 ext. 103, Fax +62 (0) 341 - 417634

Email: m.wartana@lecturer.itn.ac.id

\section{INTRODUCTION}

The ever-increasing demand for electrical energy use must be met by increasing the capacity, security, and stability of power systems, including the processes of generation, transmission, and distribution. One way to simultaneously increase the power generation capacity and improve the performance of a power system is through the appropriate integration of distributed generators (DGs) into the grid system [1], [2]. In addition, the optimal installation of a flexible alternating current transmission system (FACTS) can improve the network, the voltage profile, and the equality, efficiency, and reliability of the system, and can reduce system power losses.

The optimal integration of DG units and FACTS controllers into the grid system can play a significant role in improving system performance, by reducing power loss, increasing the voltage profile, and increasing system reliability [3]. However, installing these devices in an unsuitable location and with inappropriate sizes can produce negative impacts, such as increased power losses and violations of system 
constraints. Determining the extent to which a given type of DG, such as a wind turbine generator (WTG), can be integrated into the grid while keeping the power system safe is a time-consuming and challenging task. Several issues need to be considered, such as the long and short-term transient stability of the rotor angle, the frequency, voltage, and critical clearing time, and small signal stability analyses of the local, interarea, torque, and control modes [4], [5]. In order to ensure the reliability, efficiency, stability, and performance of the system, these analyses require a knowledge of various wind energy penetration limits [6].

In many previous studies, various approaches to optimizing computational intelligence have been proposed in relation to planning the placement of the DG and FACTS. An optimization search backtracking algorithm for the allocation of multi-type generators was used in [7] to improve the operating performance of a swarm optimization technique, in the context of determining the location and optimal size of the DG. In [8], taking into account the costs due to operating risk, a method of estimating points to determine the optimal allocation of DGs in distribution systems was also proposed. Studies focusing on the optimal allocation of multi-type DGs (PV and WTGs) have also been carried out to minimize power loss in a power network [9]. In [10], the optimal allocation of new DGs with a FACTS controller was proposed, with the aim of reducing electric power loss using genetic algorithms. Furthermore, a multi-objective tabu search algorithm was applied to determine the location and size of several types of FACTS and DGs in a power system network [11]. The majority of recent articles have focused on improving optimization procedures by applying different optimization techniques, but have used less practical test systems.

In this paper, the optimal integration of one type of DG (wind energy, WE) and a Shunt-FACTS controller into the grid is proposed, with the aim of reducing the loss of electric power. The problem is also generalized by considering one type of Shunt-FACTS, namely a static var compensator (SVC), including the use of hybrid solutions, such as installing the WE type doubly-fed induction generator (DFIG) and SVC. These can enhance the voltage profile, whereas a reduction in power loss due to improved system performance is achieved by maximizing the load bus (MLB) system scenario. Newton-Raphson power flow with DFIG and SVC is used to formulate a multi-purpose optimization problem, namely the MLB system, in which the system $P_{\text {loss }}$ is minimized by satisfying various system constraints, such as the loading limit, generation limit, voltage limit, and small-signal stability. Modifications to the IEEE 14 bus standard test system and the Indonesian Java-Bali 24 bus system, connected to the DFIG and SVC using PSAT software, are carried out in this study.

\section{RESEARCH METHOD}

\subsection{Wind turbine modeling}

Due to its advantages over other approaches, most wind farms use a variable-speed WTG, equipped with a doubly-fed induction generator (DFIG). The analysis of WE dynamics using this type of WTG has become an exciting research issue, particularly with regard to system stability [12]. Figure 1 shows the main components of the general structure of a WTG based on DFIG: a WT, gearbox, winding rotor induction generator, back-to-back converter, and controller [13]. The rotor and stator of the induction generator are fed via a back-to-back rotor voltage source converter, which is connected directly to the grid. In (1) gives the steady-state electrical equation used here.

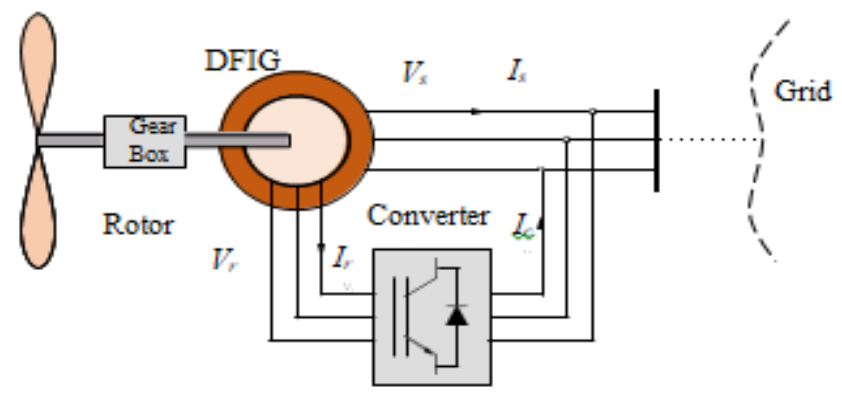

Figure 1. Variable-speed WTG with DFIG modeling

where $v_{d s}$ and $v_{q s}$ are the stator voltages on the d-and q-axes; $v_{d r}$ and $v_{q r}$ are the rotor voltages on the dand q-axes; $i_{d s}$ and $i_{q s}$ are the stator currents on the d- and q-axes; $i_{d r}$ and $i_{q r}$ are the rotor currents on the $\mathrm{d}$ - and q-axes; $r_{S}$ and $r_{R}$ are the resistances of the stator and rotor; $x_{S}$ and $x_{R}$ are the reactances of the 
stator and rotor; and $x_{m}$ and $\omega_{m}$ are the magnetization reactance and the rotor speed. Both $v_{d s}$ and $v_{q s}$ are functions of the grid magnitude and phase and are given by,

$$
\left.\begin{array}{l}
v_{d s}=-r_{s} i_{d s}+\left(\left(x_{S}+x_{m}\right) i_{q s}+x_{m} i_{q r}\right) \\
v_{q s}=-r_{s} i_{q s}+\left(\left(x_{S}+x_{m}\right) i_{d s}+x_{m} i_{d r}\right) \\
v_{d r}=-r_{R} i_{d r}+\left(1-\omega_{m}\right)\left(\left(x_{R}+x_{m}\right) i_{q r}+x_{m} i_{q s}\right) \\
v_{q r}=-r_{R} i_{q r}-\left(1-\omega_{m}\right)\left(\left(x_{R}+x_{m}\right) i_{d r}+x_{m} i_{d s}\right)
\end{array}\right\}
$$

the active and reactive power of the generator depends on the stator and current converter, as shown in (3),

$$
\left.\begin{array}{l}
P=v_{d s} i_{d s}+v_{q s} i_{q s}+v_{d c} i_{d c}+v_{q c} i_{q c} \\
Q=v_{q s} i_{d s}-v_{d s} i_{q s}+v_{q c} i_{d c}-v_{d c} i_{q c}
\end{array}\right\}
$$

\subsection{Static var compensator (SVC) modeling}

One popular type of FACTS is the SVC, which acts as a shunt-connected variable reactor, and can inject or absorb reactive power to regulate the voltage connected to the bus. This device provides instant reactive power for voltage support, and has two capacitive and inductive regions. The SVC can inject reactive and inductive power in either the capacitive and inductive mode [14]. Figure 2 shows an SVC equivalent circuit, which can be modeled as a susceptance variable depending on the particular node's requirements. In this model, the differential (4) and algebraic (5) give the total reactance of $b_{S V C}$ and the reactive power injected at the SVC node [15],

$$
\begin{aligned}
& \dot{b}_{S V C}=\left(K_{r}\left(V_{r e f}+v_{P O D}-V\right)-b_{S V C}\right) / T_{r} \\
& Q=b_{S V C} V^{2}
\end{aligned}
$$

where $K_{r}$ and $T_{r}$ are the regulator gain and regulator time constant, respectively, and $V_{r e f}$ is the reference voltage. Figure 2 shows the SVC model, which is assumed to be a time-constant regulator.

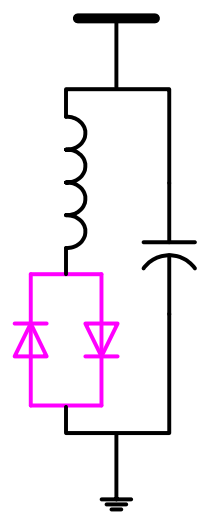

(a)

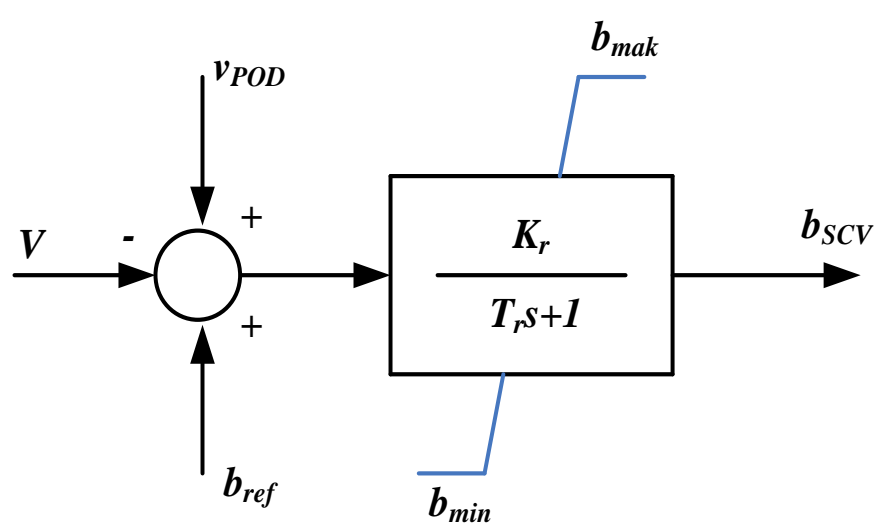

(b)

Figure 2. (a) SVC model in power system; (b) block diagram model of SVC 
where $b_{\max }$ and $b_{\min }$ are the maximum and minimum susceptance (p.u), and $v_{P O D}$ is the input signal for power system oscillation damping.

\subsection{Problem formulation}

With the scenario of increasing load achieving MLB system but with minimum line power loss $\left(P_{\text {loss }}\right)$, including different conflicts, two optimization objectives are chosen to validate the optimization algorithm developed when calculating overhead remains minimal. This multi-purpose can be simultaneously handled by NSGA-II, which is enhanced as long as it can reveal it while maintaining security and system stability. Based on a discrete-continuous mixed multi-purpose optimization with real constrained $f(\mathbf{x}, \mathbf{u})$, the problem can be formulated, as shown in (6) [16]. The dependent and control variables are represented by $\mathrm{x}$ and $\mathrm{u}$, respectively.

$$
\begin{aligned}
& \text { Minimize } f(\mathbf{x}, \mathbf{u})=\left[-f_{1}(\mathbf{x}, \mathbf{u}), f_{2}(\mathbf{x}, \mathbf{u})\right] \\
& \text { Subject to : } \begin{cases}g_{i}(\mathbf{x}, \mathbf{u})=0 & i=1, \ldots \ldots ., M \\
h_{j}(\mathbf{x}, \mathbf{u}) \leq 0 & j=1, \ldots \ldots, N\end{cases}
\end{aligned}
$$

Where $f_{l}$ and $f_{2}$ are the objective functions to be optimized, and $g_{i}$ and $h_{j}$ are the $i^{\text {th }}$ and $j^{\text {th }}$ inequality constraints, respectively. $M$ and $N$ are the numbers of equality and inequality constraints, respectively.

\subsection{Maximizing load system}

The first objective function of this research is the MLB system, with a load increase scenario as shown on,

$$
\begin{aligned}
& \text { Maximize } f_{1}(\mathbf{x}, \mathbf{u}, \lambda) \\
& \text { Subject to : } V L=\sum_{i=1}^{N_{L}} O L L_{i}+\sum_{j=1}^{N_{E}} B V V_{j}
\end{aligned}
$$

where $\lambda$ is the system load parameter, derived in (10), and $V L$, which is the sum of $O L L_{i}$ and $B V V_{j}$ (shown in (13) and (14), respectively), represents the thermal and bus violation limit factors. $N_{L}$ and $N_{E}$ are the total numbers of transmission lines and load buses, respectively [17].

$$
\lambda=\exp \left[\gamma\left|\lambda_{f}-\lambda_{f}^{\max }\right|\right] ; \quad \lambda_{f} \in\left[1, \lambda_{f}^{\max }\right]
$$

Where $\gamma$ is the slope adjustment coefficient of the function, $P_{D i}$ and $Q_{D i}$ are the active and reactive power demands (as shown in (11) and (12), respectively), and the load factor $\lambda_{f}$, has a maximum value of $\lambda f^{\max }$.

$$
\begin{aligned}
& P_{D i}\left(\lambda_{f}\right)=\lambda_{f} P_{D i} \\
& Q_{D i}\left(\lambda_{f}\right)=\lambda_{f} Q_{D i}
\end{aligned}
$$

The first term in (9), $O L L_{i}$, which is defined in (13), represents the system security state's indices, and its value is equal to one if the $j^{\text {th }}$ line loading is less than its rating. Otherwise, it increases logarithmcally with the overload, as shown,

$$
O L L_{i}=\left\{\begin{array}{lll}
1 ; & \text { if } & P_{i j} \leq P_{i j}^{\max } \\
\exp \left(\Gamma_{O L L}\left|1-\frac{P_{i j}}{P_{i j}^{\max }}\right|\right) ; & \text { if } & P_{i j} \geq P_{i j}^{\max }
\end{array}\right.
$$

where $P_{i j}$ and $P_{i j}^{\max }$ are the real power flows between buses $i$ and $j$ and their thermal limit. The coefficient 
used to adjust the slope of the exponential function is $\Gamma_{O L L}$. The second term, $B V V_{j}$, defined in (9), gives the system security indices that are associated with the bus voltage violation factor for bus $j$, as shown in (14),

$$
B V V_{j}= \begin{cases}1 ; & \text { if } \quad 0,9 \leq \mathrm{v}_{\mathrm{b}} \leq 1.1 \\ \exp \left(\Gamma_{B V V}\left|1-V_{b}\right|\right) ; & \text { otherwise }\end{cases}
$$

where $\Gamma_{B V V}$ is the coefficient used to adjust the slope of the exponential function, in a similar way to (13). If $B V V_{j}$ is equal to one, the voltage level drops to between its minimum and maximum limits; otherwise, the voltage deviation increases exponentially.

\subsection{Minimizing the line power loss}

Minimizing the line power loss $\left(P_{\text {loss }}\right)$ of the transmission line is the second objective function, as formulated [18],

$$
f_{2}(\mathbf{x}, \mathbf{u})=P_{\text {loss }}=\sum_{k=1}^{n l} g_{k}\left[V_{i}^{2}+V_{j}^{2}-2 V_{i} V_{j} \cos \left(\delta_{i}-\delta_{j}\right]\right.
$$

where $n l$ is the transmission line number, $g_{k}$ is the conductance of the $k^{\text {th }}$ line; $V_{i} \delta_{i}$ is the voltage on the end bus $i$, and $V_{j} \delta_{j}$ is the voltage on the end bus $j$ of the $k^{\text {th }}$ line.

\subsection{Equality and inequality constraints}

\subsubsection{Equality constraints}

The typical load flow equations are denoted as their equality constraints expressed in (16),

$$
\left.\begin{array}{ll}
P_{G_{i}}=P_{L_{i}}+V_{i} \sum_{i=1}^{N_{b}} V_{j}\left(G_{i j} \cos \delta_{i j}+G_{i j} \sin \delta_{i j}\right) ; & i=1,2, \ldots \ldots, N_{b} \\
Q_{G_{i}}=Q_{L_{i}}+V_{i} \sum_{i=1}^{N_{b}} V_{j}\left(G_{i j} \sin \delta_{i j}+G_{i j} \cos \delta_{i j}\right) ; & i=1,2, \ldots \ldots, N_{b}
\end{array}\right\}
$$

where $N_{b}$ is the number of buses.

\subsubsection{Inequality constraints}

Active and reactive power generators $P_{G i}$ and $Q_{G i}$, respectively, voltage $V_{i}$, and phase angle $\delta_{i}(17)$. The parameter settings SVC, $b_{S V C}$ in (18) and transmission loading $P_{i j}$ at (19) represents inequality constraints $h_{j}(\mathbf{x}, \mathbf{u})$ in $(15)$, whose value is limited by their limits,

$$
\left.\begin{array}{lr}
P_{G_{i}}^{\min } \leq P_{G_{i}} \leq P_{G_{i}}^{\max } ; & i=1,2, \ldots \ldots ., m \\
Q_{G_{i}}^{\min } \leq Q_{G_{i}} \leq Q_{G_{i}}^{\max } ; & i=1,2, \ldots \ldots ., m \\
V_{i}^{\min } \leq V_{i} \leq V_{i}^{\max } ; & i=1,2, \ldots \ldots ., m \\
-0,9 \leq \delta_{i} \leq 0,9 ; & i=1,2, \ldots \ldots ., m
\end{array}\right\}
$$

\subsection{Stability constraints}

\subsubsection{Small-signal stability}

One of the stability indices that is applied to improve system performance in this study is the small-signal stability. This power system stability index reflects the system's ability to return to regular or stable operation after several minor disturbances, as indicated by the eigenvalues of the system matrix, which characterize the stability of the system [19]. The increase in system loading (MLB) system and a large injection of WT into the grid can affect the stability of the distribution and transmission system [4], mainly due to the nonlinear dynamic behavior of WTG [5]. In (20) gives a set of differential algebraic equations (DEAs) that are used for small signal stability analysis, 


$$
\left.\begin{array}{l}
\dot{x}=f(x, y) \\
0=g(x, y)
\end{array}\right\}
$$

where $\mathrm{x}$ and $\mathrm{y}$ denote the state vector and the set of algebraic variables, respectively. To calculate the state matrix $A_{s}$, we use the complete Jacobian matrix manipulation $A_{C}$ by determining the linearization of the DAE system (21) [20],

$$
\left[\begin{array}{c}
\Delta \dot{x} \\
0
\end{array}\right]=\left[\begin{array}{ll}
\nabla_{x} f & \nabla_{y} f \\
\nabla_{x} g & \nabla_{y} g
\end{array}\right]\left[\begin{array}{l}
\Delta x \\
\Delta y
\end{array}\right]=\left[A_{C}\right]\left[\begin{array}{l}
\Delta x \\
\Delta y
\end{array}\right]
$$

By eliminating the algebraic variables, the status $A_{s}$ of the matrix is obtained, as shown in (22). This expression implicitly assumes that there are no singularity-induced bifurcations [15],

$$
A_{S}=F_{x}-F_{y} G_{y}^{-1} G_{x}
$$

when this matrix has been obtained, we can calculate the eigenvalues in the S-domain. If the real part of the eigenvalues is less than zero, then the system is stable.

\subsubsection{Fast voltage stability index}

One of the stability indices used to ensure safe bus loading in this study is the fast voltage stability index (FVSI) [21], as defined,

$$
F V S I_{i j}=\frac{4 Z^{2} Q_{j}}{V_{i}^{2} X}
$$

if the value of FVSI is close to 1.00 , this indicates that the line is approaching the point of instability, and if the amount exceeds 1.00 , a sudden voltage drop can occur on one of the buses connected to the line, causing the system to collapse.

\subsubsection{Line stability factor}

In (24) gives an expression for the line stability factor (LQP), which is applied to [22] to ensure the system stability index if the value is less than 1.00 ,

$$
L Q P_{i j}=4\left(\frac{X}{V_{i}^{2}}\right)\left(\frac{X}{V_{i}^{2}} P_{i}^{2}+Q_{j}\right)
$$

\subsection{Brief description of NSGA-II}

The technique used to solve the optimization problem (MO) is a variant of the non-dominated-based genetic algorithm, and is called the non-dominated sorting genetic algorithm II (NSGA-II) [23]. The process of the NSGA II algorithm can be summarized. First, the population is initialized and sorted according to the objective function, based on the non-domination of each front. Each Pareto front and individual is then ranked sequentially, based on the non-domination criterion. The first front and individuals who dominate others are assigned rank 1. Furthermore, the second front dominates the others except for the first front ranks second and so on. The crowded distance approach is applied to other members of the same Pareto front, with the same non-dominant rank are then given a distance whose value is assigned to individuals in the same Pareto front [24]. Finally, the parents and offspring are combined to form a population, and future generations are selected from this population as described in [25]. To find the best solution from the set of possible solutions that meet the conflicting objectives of the Pareto front, a fuzzy set with full membership is considered the best compromise solution (CS) [25].

\section{RESULTS AND DISCUSSION}

Several scenarios were modeled to prove the efficacy of the proposed approach, using both a modified IEEE 14-bus standard test system [26], [27] and a practical test system, which in this case was the Indonesia Java-Bali 24-bus system [22]. Furthermore, one type of wind field as WTG, DFIG, can transmit large amounts of active power to the system and consumes and produces reactive power. The shunt-FACTS 
control system, SVC, is optimally installed on the grid to control the system's stability and security due to the MLB system. In order to investigate the MLB system while minimizing $P_{\text {loss }}$ by maintaining various security and stability systems in the integrated WTG based DFIG with SVC controllers into the grid, a simulation based on NSGA-II was developed, for several scenarios: (a) the base case, without WTG or SVC; (b) Scenario 1, with WTG only; (c) Scenario 2, with SVC only; and (d) Scenario 3, with WTG and SVC.

\subsection{IEEE 14-bus system}

\subsubsection{Base case: without WTG or FACTS controllers}

With the NSGA-II technique in the base case condition, the grid is not connected to WTG and SVC. A Pareto front is obtained as shown in Figure 3. It can be seen from the figure that the MLB system and the minimum $P_{\text {loss }}$ were $149.59 \%$ and 0.1625 p.u, respectively. However, the best CS, although obtained by $P_{\text {loss }}$, was slightly lower than the previous result of 1.1704 p.u, but with a meager MLB system of only $114.40 \%$. In this case, all system stability is not considered.

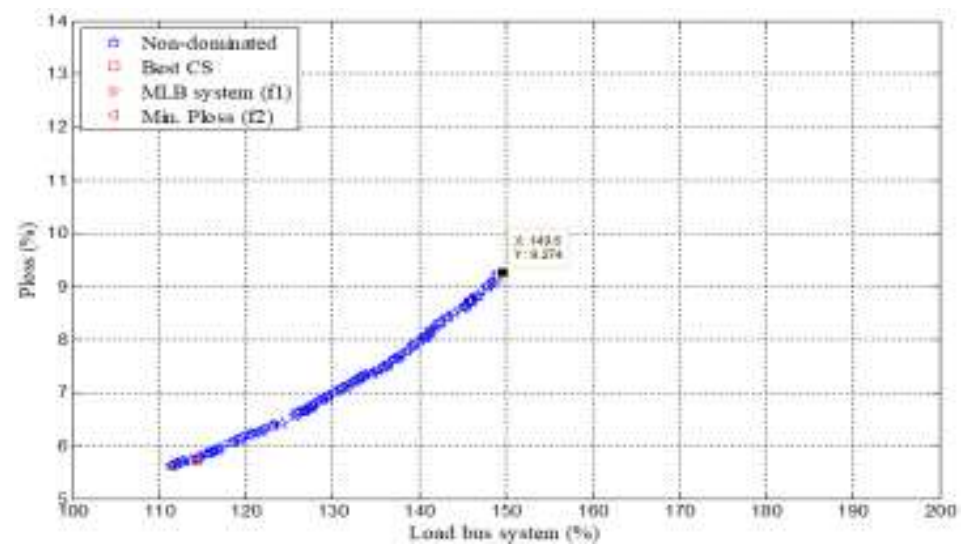

Figure 3. Pareto front for the base case

\subsubsection{Scenario 1: WTG only}

Figure 4 shows the best CS from the placement of WTG on bus 8, with active and reactive power capacities of $49.91 \mathrm{MW}$ and -11.56 MVAr, respectively. In this case, all the stability limits are satisfied. Meanwhile, the placement of WTG on the same bus produces the best $P_{\text {loss }}$ of $0.1772 \mathrm{p} . \mathrm{u}$, although it can only improve the MLB system 112.24\%, which is the lowest in Scenario 1. An MLB system of $157.08 \%$ and $P_{\text {loss }}$ of 0.4885 p.u were also obtained when installing the WTG on bus 14 ; these were the highest values in this scenario. These results are more extensive than the results obtained for the base scenario. The system stability in the form of a small signal on the best CS, stated by the S field's negative eigenvalues, is shown in Figure 5. This value proves that installation of the WTG at the best location guarantees the stability of the grid system. The graphs in the figure only include real eigenvalues of less than -3 .

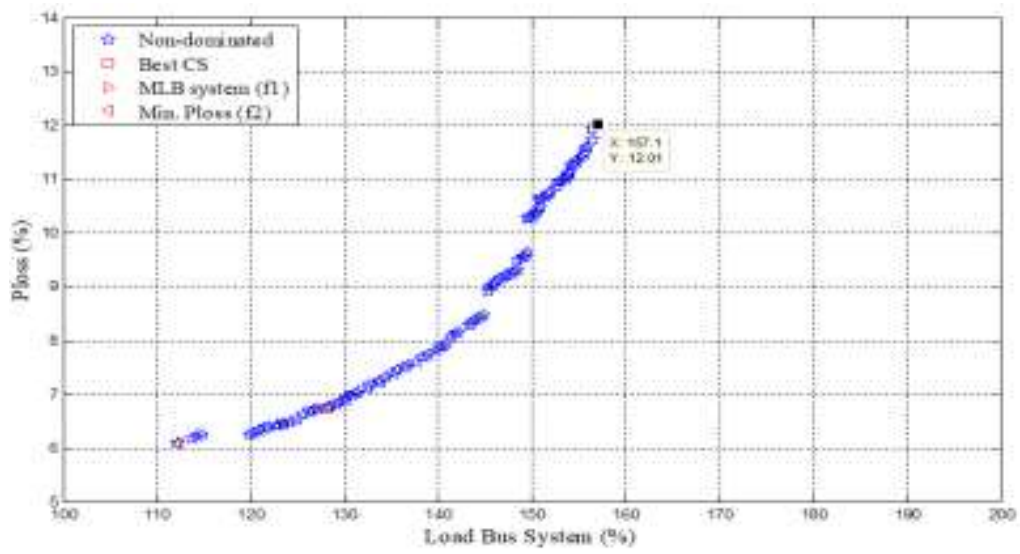

Figure 4. Pareto front for Scenario 1 


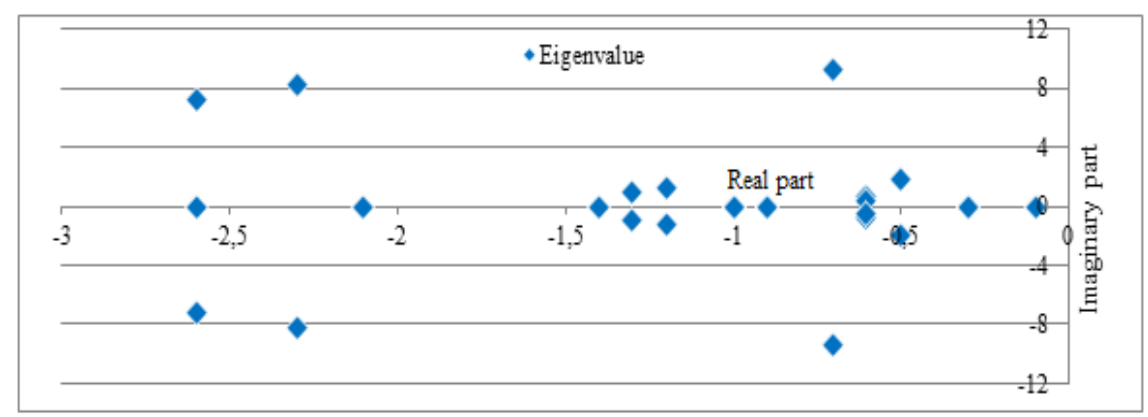

Figure 5. Eigenvalues for Scenario 1

\subsubsection{Scenario 2: SVC only}

Figure 6 illustrates the best solution obtained by placing the SVC on buses 9 and 5, with settings of 0.01 and 1.09 p.u. The placement of the shunt-FACTS on these two buses provides the best MLB system and the best $P_{\text {loss }}$, with values of $181.64 \%$ and 0.5562 p.u., respectively. The best value obtained for $P_{\text {loss }}$ was slightly higher than that obtained in Scenario 1. Meanwhile, the best CS from the MLB systems and $P_{\text {loss }}$ were $124.16 \%$ and 0.2041 p.u, respectively, with the optimal placement of the SVC on bus 13 with a setting of 0.2529 ; this satisfies the small-signal stability constraint, as shown in Figure 7.

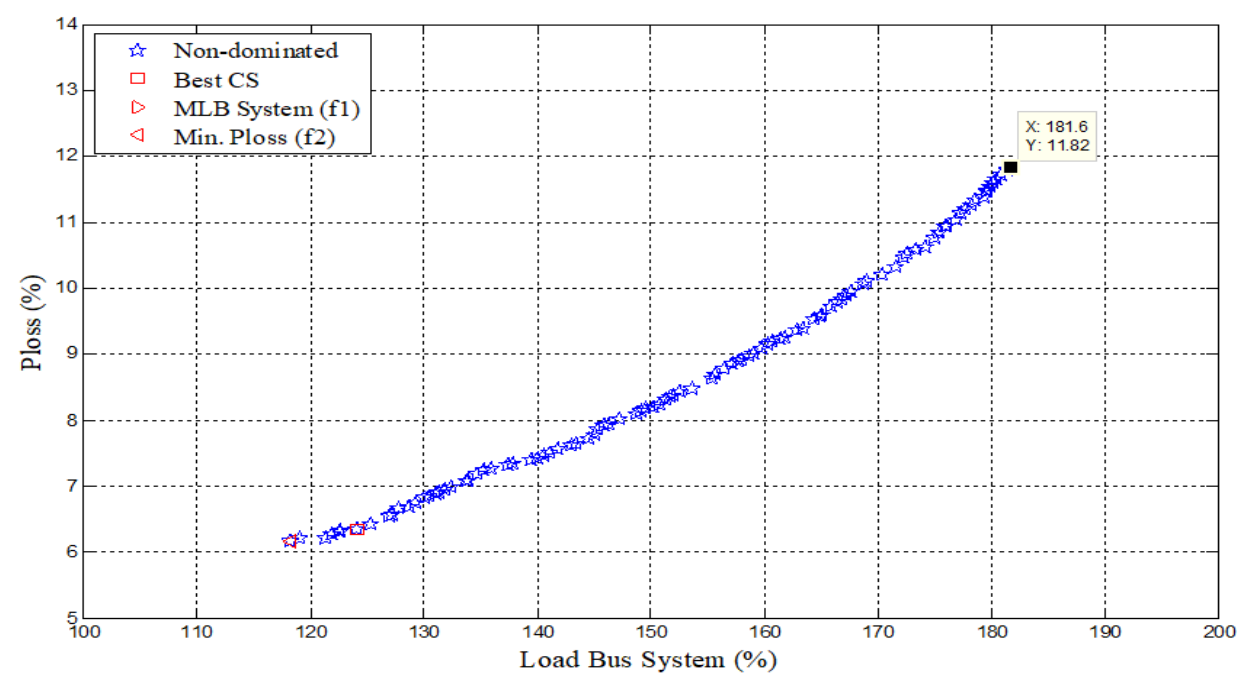

Figure 6. Pareto front for Scenario 2

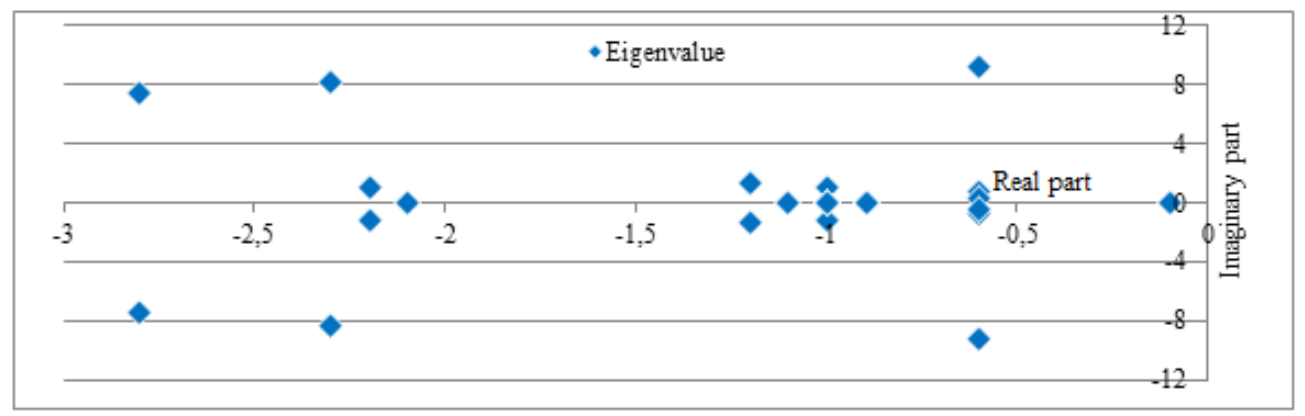

Figure 7. Eigenvalues for Scenario 2 


\subsubsection{Scenario 3: WTG and SVC}

A summary of the extreme points of the optimal solution is shown in Figure 8, with the optimal placement of WTG on bus 3 with active and reactive power respectively 52.93 MW and -23.20 MVAr and installing SVC on bus 7 with a setting of 0.6144. p.u obtained the best CS with the MLB system was $126.25 \%$ and $P_{\text {loss }} 0.2429$ p.u. The figure also shows that the installation of the SVC on buses 9 and 5 with a location with the WTG location in the same optimal place, namely on bus 4, gives the best MLB system and $P_{\text {loss }}$ systems, with values of $182.79 \% 0.2167$ p.u, respectively. The best CS results are higher than for Scenarios 1 and 2. Figure 9 depicts the eigenvalues and shows that the system is stable under all conditions. Figures 10 and 11 show the voltage and line stability indices, and it can be seen that for all scenarios, the FVSI and LPQ have values of less than 1.00. This condition ensures that no lines will be overloaded and that no buses will collapse due to overloading.

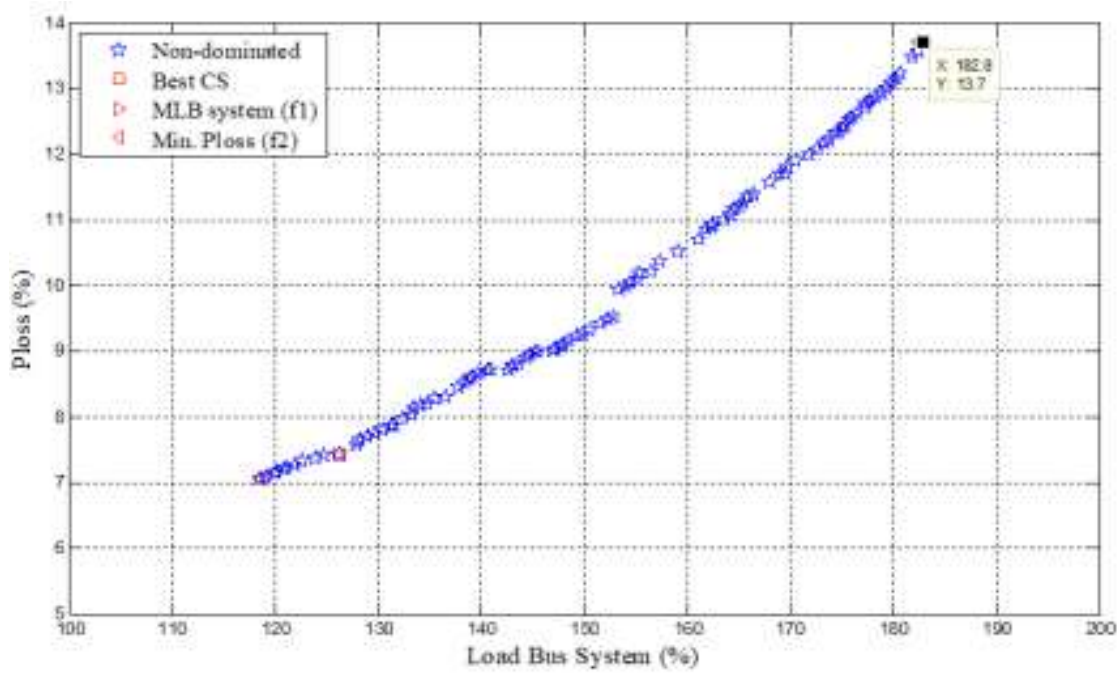

Figure 8. Pareto front for Scenario 3

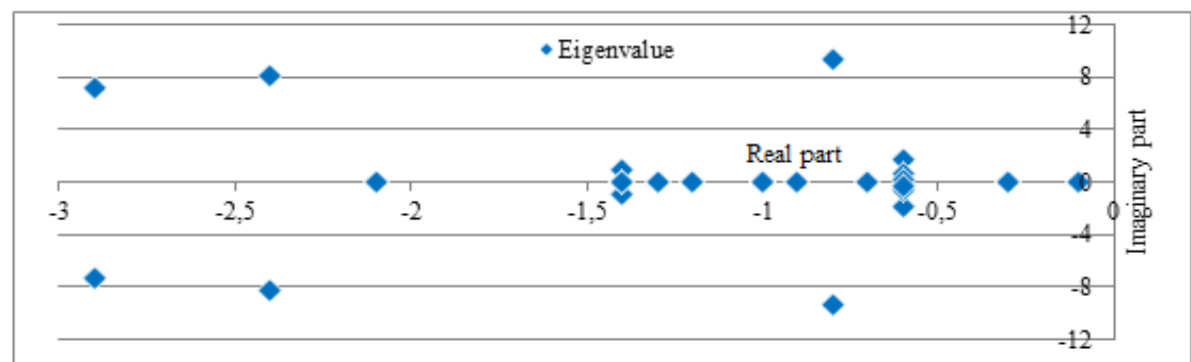

Figure 9. Eigenvalues for Scenario 3

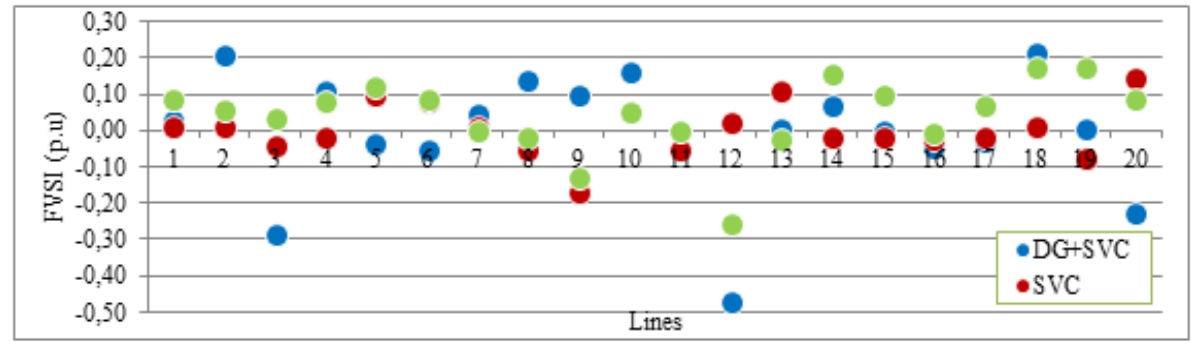

Figure 10. FVSI for all scenarios 


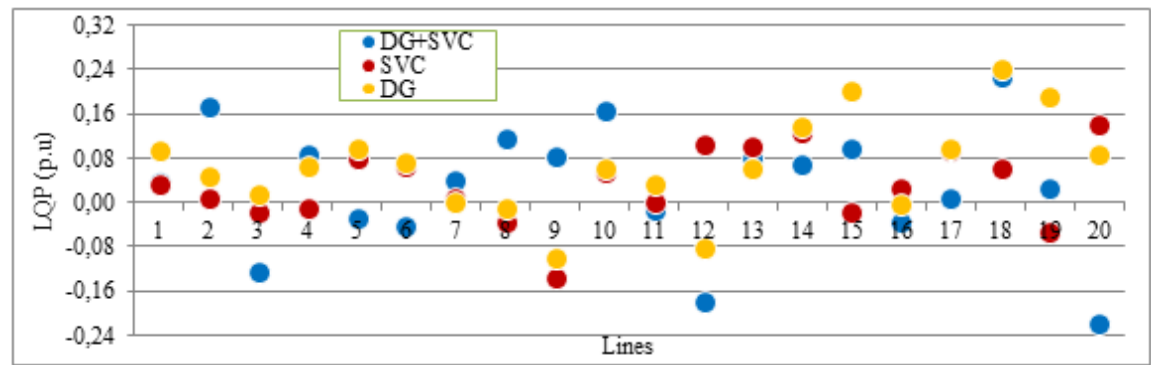

Figure 11. LQP for all scenarios

\subsection{Indonesia Java-Bali 24-bus system}

The method developed here was also successfully tested on the Indonesian Java-Bali 24-bus practical test system [20] for all the scenarios described above. Figure 12 presents the Pareto front results for Scenario 3, and it can be seen that the optimal placement of the SVC is on bus 16 with a setting of 0.6144 p.u. and the installation of the WTG in the best location on bus 13 with a size of $61.64 \mathrm{MW}$ and -22.39 MVar gives the optimal MLB system and $P_{\text {loss }}$ of $130.63 \%$ and $1.205 \%$, respectively for the best CS results. From the same figure, it can be observed that with the integration of WTG on bus 13 with a capacity of 61.64 MW and -22.39 MVar and the SVC installation on bus 19 with a setting of 0.9128 p.u, the MLB system reaches $158.54 \%$, and the value of $P_{\text {loss }}$ obtained is $2,327 \%$.

The eigenvalues shown in Figure 13 prove that the system is stable under all conditions. Simultaneously, the FVSI and LPQ indices for the practical test system in all scenarios are shown in Figures 14 and 15, respectively. The results prove that the system is stable in the MLB system.

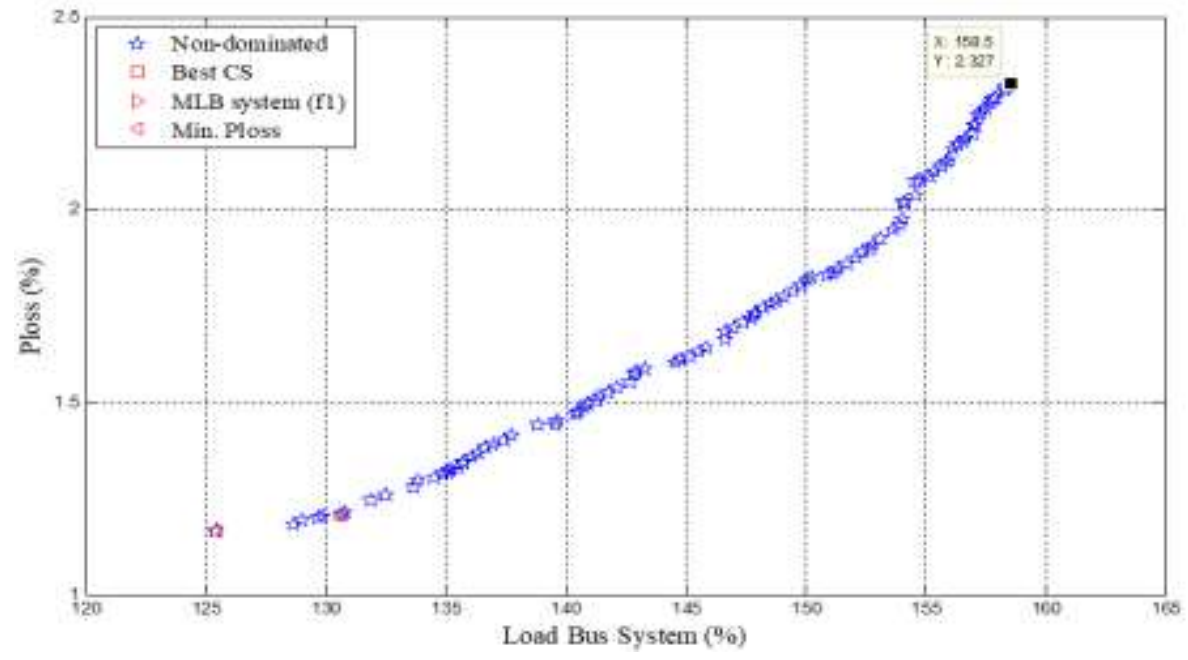

Figure 12. Pareto front for Scenario 3, for the Indonesia Java-Bali 24-bus system

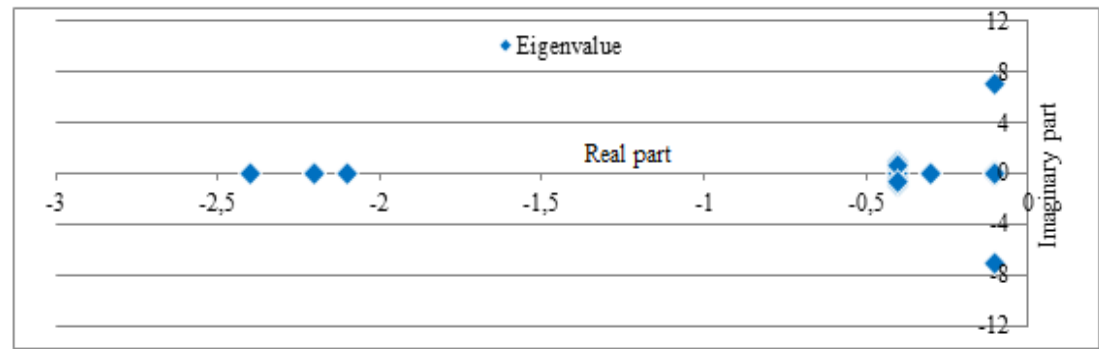

Figure 13. Eigenvalues for Scenario 3, for the Indonesia Java-Bali 24-bus system 


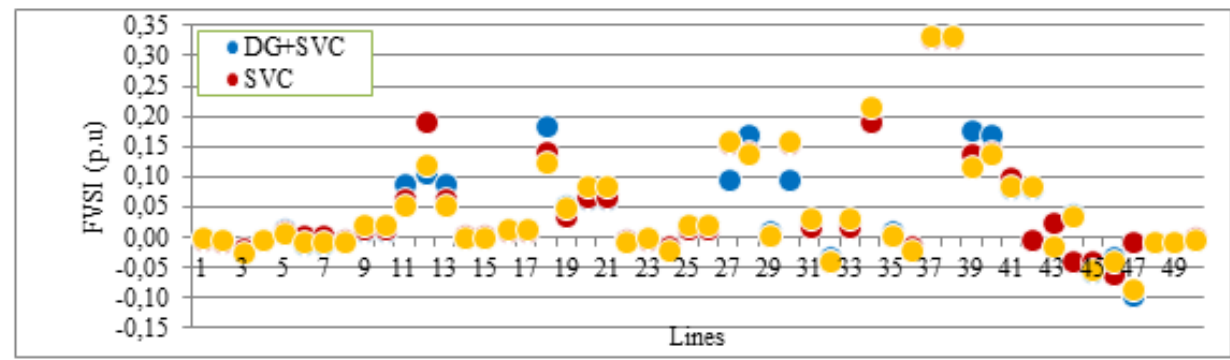

Figure 14. FVSI for all scenarios, for the Indonesia Java-Bali 24-bus system

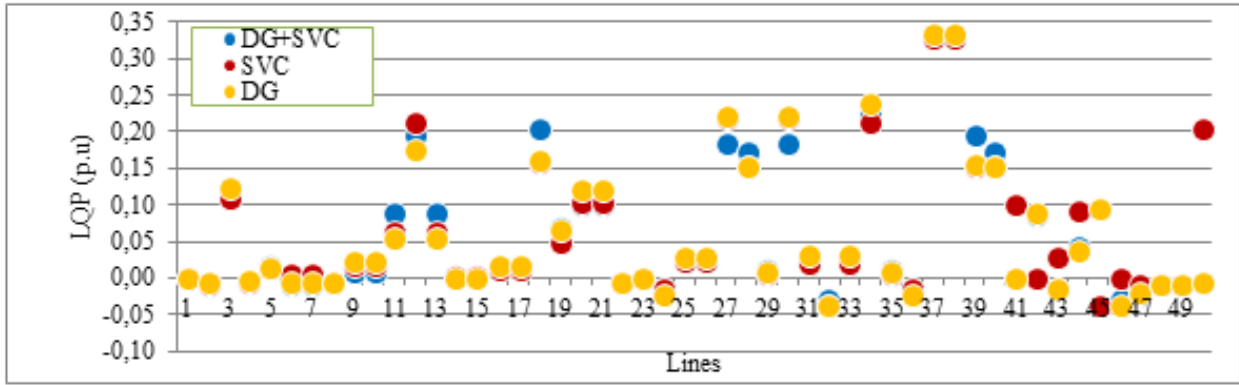

Figure 15. LQP for all scenarios, for the Indonesia Java-Bali 24-bus system

\section{CONCLUSION}

In this study, a multi-objective problem was addressed that involved increasing the system loading (MLB) while reducing the power loss $\left(P_{\text {loss }}\right)$, via the optimal placement of a WTG (wind farm) and SVC on the grid using NSGA-II. The main objective was to improve the system performance at the margin of safety and stability, within the allowable limits. This conflicting bi-objective optimization problem was tested on the IEEE 14-bus system test standard and practical strategies, based on two system safety status indices: the line overload limit and the bus voltage violation limit. To improve the practicality of the proposed solution, various system stability constraints, such as the small-signal stability, FVSI, and LQP, were evaluated for each scenario placement, adjustment, and determination of the SVC's optimal size and WTG. Using the crowding distance technique, the Pareto optimal solution's front side can be maintained. Meanwhile, to quote the best CS from the Pareto front is done by applying the fuzzy-based mechanism.

\section{ACKNOWLEDGEMENTS}

The authors would like to express their gratitude and appreciation to the Ministry of Research and Technology of the Republic of Indonesia, for supporting the funding of this research through contract agreement Number: 053/ SP2H /LT-MULTI/L7/2020 (Mar 17, 2020), and through the ITN research and community service institutions, Malang number: ITN .03.0335.8 / IX.REK / 2020 (Mar 18, 2020).

\section{REFERENCES}

[1] Z. Liu, F. Wen, and G. Ledwich, "Optimal siting and sizing of distributed generators in distribution systems considering uncertainties," IEEE Transactions on Power Delivery, vol. 26, no. 4, pp. 2541-2551, 2011, doi: 10.1109/TPWRD.2011.2165972.

[2] P. Dehghanian, S. H. Hosseini, M. Moeini-Aghtaie, and A. Arabali, "Optimal siting of DG units in power systems from a probabilistic multi-objective optimization perspective," International Journal of Electrical Power \& Energy Systems, vol. 51, pp. 14-26, 2013, doi: 10.1016/j.ijepes.2013.02.014.

[3] Eladl, A. Elmitwally, S. Eskander, and I. Mansy, "Optimal allocation of FACTS devices in restructured power systems integrated wind deneration," Bulletin of the Faculty of Engineering. Mansoura University, vol. 40, no. 1, pp. 26-41, 2020, doi: 10.21608/bfemu.2020.100769.

[4] T. Adachi and A. Yokoyama, "Improvement of small-signal stability of power system by controlling doubly fed induction generators of a large-capacity wind farm," Journal of International Council on Electrical Engineering, vol. 6, no. 1, pp. 117-125, 2016, doi: 10.1080/22348972.2016.1181701. 
[5] J. Bhukya and V. Mahajan, "Integration of DFIG based wind turbine generator on small signal stability of power systems," in 2017 Innovations in Power and Advanced Computing Technologies (i-PACT): IEEE, pp. 1-6, 2017, doi: 10.1109/IPACT.2017.8244971.

[6] R. Krishan, A. Verma, and B. Prasad, "Small signal stability analysis of grid connected distributed PV and wind energy system," in 2014 6th IEEE Power India nternational Conference (PIICON): IEEE, pp. 1-6, 2014, doi: 10.1109/POWERI.2014.7117730.

[7] El-Fergany, "Optimal allocation of multi-type distributed generators using backtracking search optimization algorithm," International Journal of Electrical Power \& Energy Systems, vol. 64, pp. 1197-1205, 2015, doi: 10.1016/j.ijepes.2014.09.020.

[8] Q. Gong, J. Lei, and J. Ye, "Optimal siting and sizing of distributed generators in distribution systems considering cost of operation risk," Energies, vol. 9, no. 1, p. 61, 2016, doi: 10.3390/en9010061.

[9] B. Ahmadi, O. Ceylan, and A. Ozdemir, "Optimal allocation of multi-type distributed generators for minimization of power losses in distribution systems," in 2019 20th International Conference on Intelligent System Application to Power Systems (ISAP): IEEE, pp. 1-6, 2019, doi: 10.1109/ISAP48318.2019.9065974.

[10] S. Burana, P. Thararak, P. Jirapong, and K. Mansuwan, "Optimal allocation of distributed generation with facts controller for electrical power loss reduction using genetic algorithm," in 2017 9th International Conference on Information Technology and Electrical Engineering (ICITEE): IEEE, pp. 1-6, 2017, doi: 10.1109/ICITEED.2017.8250468.

[11] O. A. Coronado de Koster and J. A. Domínguez-Navarro, "Multi-objective tabu search for the location and sizing of multiple types of FACTS and DG in electrical networks," Energies, vol. 13, no. 11, p. 2722, 2020, doi: 10.3390/en13112722.

[12] B. Mehta, P. Bhatt, and V. Pandya, "Small signal stability analysis of power systems with DFIG based wind power penetration," International Journal of Electrical Power \& Energy Systems, vol. 58, pp. 64-74, 2014, doi: 10.1016/j.ijepes.2014.01.005.

[13] C. Zhang, D. Ke, Y. Sun, C. Chung, J. Xu, and F. Shen, "Coordinated supplementary damping control of DFIG and PSS to suppress inter-area oscillations with optimally controlled plant dynamics," IEEE Transactions on Sustainable Energy, vol. 9, no. 2, pp. 780-791, 2017, doi: 10.1109/TSTE.2017.2761813.

[14] V. Suresh and S. Sreejith, "Power flow analysis incorporating renewable energy sources and FACTS devices," International Journal of Renewable Energy Research (IJRER), vol. 7, no. 1, 2017, doi: 10.1234/IJRER.V7I1.5074.

[15] F. Milano, L. Vanfretti, and J. C. Morataya, "An open source power system virtual laboratory: The PSAT case and experience," IEEE Transactions on Education, vol. 51, no. 1, pp. 17-23, 2008, doi: 10.1109/TE.2007.893354.

[16] M. Wartana and A. Ni Putu, "Application of Voltage and Lines Stability Index for Optimal Placement of Wind Energy with a System Load Increase Scenario," Current Alternative Energy, vol. 3, no. 1, pp. 44-49, 2019, doi: $10.2174 / 2405463103666190724105814$.

[17] M. Wartana, J. G. Singh, W. Ongsakul, and S. Sreedharan, "Optimal placement of FACTS controllers for maximising system loadability by PSO," International Journal of Power and Energy Conversion, vol. 4, no. 1, pp. 9-33, 2013, doi: 10.1504/IJPEC.2013.050931.

[18] M. El-Azab, W. Omran, S. Mekhamer, and H. Talaat, "A probabilistic multi-objective approach for FACTS devices allocation with different levels of wind penetration under uncertainties and load correlation," International Journal of Electrical and Computer Engineering, vol. 10, no. 4, p. 3898, 2020, doi: 10.11591/ijece.v10i4.pp3898-3910.

[19] P. Dey, A. Bhattacharya, and P. Das, "Tuning of power system stabilizer for small signal stability improvement of interconnected power system," Applied Computing and Informatics, 2017, doi: 10.1016/j.aci.2017.12.004.

[20] M. Wartana, N. P. Agustini, and J. G. Singh, "Optimal integration of the renewable energy to the grid by considering small signal stability constraint," International Journal of Electrical and Computer Engineering (IJECE), vol. 7, no. 5, pp. 2329-2337, 2017, doi: 10.11591/ijece.v7i5.pp2329-2337.

[21] N. P. Agustini, L. M. Hayusman, T. Hidayat, and I. M. Wartana, "Security and stability improvement of power system due to interconnection of DG to the grid," in Proceedings of Second International Conference on Electrical Systems, Technology and Information 2015 (ICESTI 2015): Springer, pp. 227-237, 2016, doi.org/10.1007/978-981287-988-2_24.

[22] M. Wartana, "A multi-objective problems for optimal integration of the DG to the grid using the NSGA-II," in 2015 International Conference on Quality in Research (QiR): IEEE, pp. 106-110, 2015, doi: 10.1109/QiR.2015.7374906.

[23] Deb, "A fast elitist non-dominated sorting genetic algorithm for multi-objective optimization: NSGA-2," IEEE Trans. Evol. Comput., vol. 6, no. 2, pp. 182-197, 2002, doi.org/10.1007/3-540-45356-3_83.

[24] P. E. Nicholas, K. Padmanaban, and M. L. Babu, "Multi-objective optimization of laminated composite plate with diffused layer angles using non-dominated sorting genetic algorithm (NSGA-II)," Advanced Composites Letters, vol. 23, no. 4, p. 096369351402300403 , 2014, doi: $10.1177 \% 2$ F096369351402300403.

[25] Mahesh, P. Nallagownden, and I. Elamvazuthi, "Advanced Pareto front non-dominated sorting multi-objective particle swarm optimization for optimal placement and sizing of distributed generation," Energies, vol. 9, no. 12, p. 982, 2016, doi: 10.3390/en9120982.

[26] F. Milano, "An open source power system analysis toolbox," IEEE Transactions on Power Systems, vol. 20, no. 3, pp. 1199-1206, 2005, doi: 10.1109/TPWRS.2005.851911.

[27] R. D. Zimmerman, C. E. Murillo Sanchez, and R. J. Thomas, "MATPOWER: Steady-state operations, planning, and analysis tools for power systems research and education," IEEE Transactions on Evolutionary Computation, vol. 26, no. 1, pp. 12-19, 2011, doi: 10.1109/TPWRS.2010.2051168. 


\section{BIOGRAPHIES OF AUTHORS}

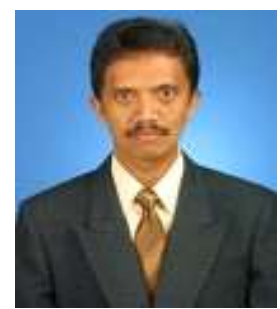

I Made Wartana received a B. Eng and an M.Eng. from the Electrical Engineering Department, National Institute of Technology (ITN) Malang, East Java, Indonesia and the Bandung Institute of Technology (ITB), West Java, Indonesia, in 1986 and 1994, respectively. He has been a lecturer at ITN Malang, Indonesia, since 1992. He also received a Dr. Eng. from the Asian Institute of Technology (AIT), Thailand, in 2012. His research interests include the application of FACTS controllers to the power grid, the application of AI to power systems, and the integration of renewable energies with the grid.

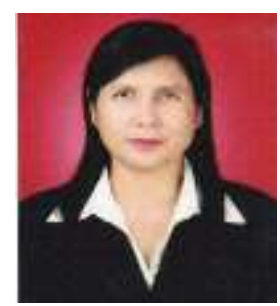

Ni Putu Agustini received a B. Eng and an M.Eng. from the Electrical Engineering Department, National Institute of Technology (ITN) Malang, East Java, Indonesia, and Brawijaya University (UB), East Java, Indonesia, in 1986 and 2008, respectively. She has been a lecturer at ITN Malang, Indonesia, since 2004. Her research interests include integrating renewable energy into the power grid and the application of reliability to power systems.

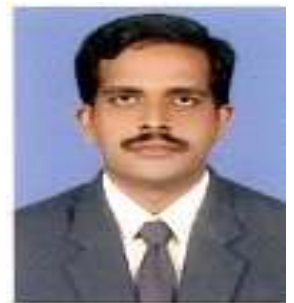

Sasidharan Sreedharan received an M. Tech in Electrical Power Systems from the Govt Engineering College, Thrissur, Kerala, India in 1998, and a Ph.D. from the Asian Institute of Technology, Bangkok, in 2010. He was a post-doctoral research fellow at the RED Lab, Renewable Energy Design Laboratory, University of Hawaii, United States, from 2014 to 2015. His research interests include the grid integration of renewable energies, the application of AI to power systems, and the stability of the grid. 